\title{
A Phenomenological Calculation of Vacuum Fluctuation in Electronic and Muonic Atoms (*)
}

\author{
B. FIICKE \\ Department of Materials Soience, Northwestern University - Evanston, Ill.
}

(ricevuto il 14 Giugno 1971)

\section{1. - Introduction.}

The experimental discovery of the I amb shift in 1947 by IAAMB and RETHERFORD ( $\left.{ }^{1}\right)$ gave rise to a quick development of quantum electrodynamies. Vacum polarization and fluctuation were calculated by different methods up to very high order in the usual expansion parameter $Z \propto$ for single-electron atoms where this effect was measured with very high accuracy $\left({ }^{2}\right)$. The noarly complete agroement between experiment and thoory in the region of very small $Z$ was a large confirmation of the methods used in quantum electrodyuanies. For high $Z$ the experimental proof of these effects is much more difficult because no single-electron atoms are available and in normal atoms there are several other effects of the same order of magnitude arising fron the many-body problem of $Z$ electrons. The effect of vacuun polarization alone can be measured with very high accuracy in muonic atoms up to uranium. It was shown recently that the calculations agree very well with experiment $\left({ }^{3}\right)$, provided that the vacuum fluctuation is very small in comparison to the vacuum polarization. This situation is opposite for electronic atoms; for example, the effect of vacuum polarization for the $1 s$ level in $\mathrm{Hg}$ is of the order of -- $3 \mathrm{Ryd}$, whereas the effect of vacuum fluctuation is of the order of +15 Ryd as given by DESIDnro et al. $\left(^{4}\right)$. BRown et al. $\left({ }^{5}\right)$ in an older calcnlation fave f-4l Ryd. The cause of this diserepancy is not known.

These computations are very complicated and are given for the 18 levels only. From this point of view it is worth-while to present a simple phenomenological calculation of the effect of vacuum fluetuation, especially for uses in self-consistent calculations as well as for the sturly of this effect in superhogy elements.

(*) Research supported by the U.S. Atomic Fnergy Commission and the Deutsche Forsehungsgeneinschaft.

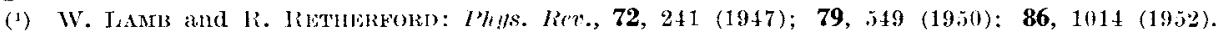

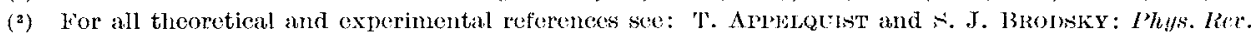
Lctt., 24, 562 (1970); and as an older roview A. l'etermans: Fontschr. I'hys., 6, 505 (1958).

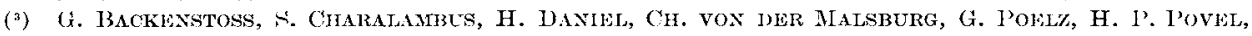
H. Schmit and I. TAuschir: Phys. Lett., $31 \mathrm{~B}, 233(1970)$ and references herein.

(i) A. M. DEsiblizio and W. IR. Jonxson: to be published in Ihys. Rev. (1971).

(5) G. F. Brown and D. F. MAYERS: Proc. Roy. Soc., A 25I, 105 (1959). 


\section{2. - The calculation of vacuum fluctuation.}

The effect of vacuum fluctuation was carly deseribed as a "zitterbewegung" of the electrons with an amplitude sr, which comes from the interaction of the electrons with the zero-point electromagnetie ficld. BJoRKeN and Driz.I. $\left({ }^{6}\right)$ give this amplitude $\delta r$ for small $Z$ as follows:

$$
\left\langle(\delta r)^{2}\right\rangle=\frac{2 \alpha}{\pi}\left(\frac{1}{m}\right)^{2} \ln \frac{1}{Z x}
$$

ir as a function of $Z$ is given in Fig. 1 as a solid line. This explanation of the effect of vacuum fluctuation was used as a simplified illustration only. But if one takes this explanation somewhat more seriously, one gets quite surprising results. A necessary con-

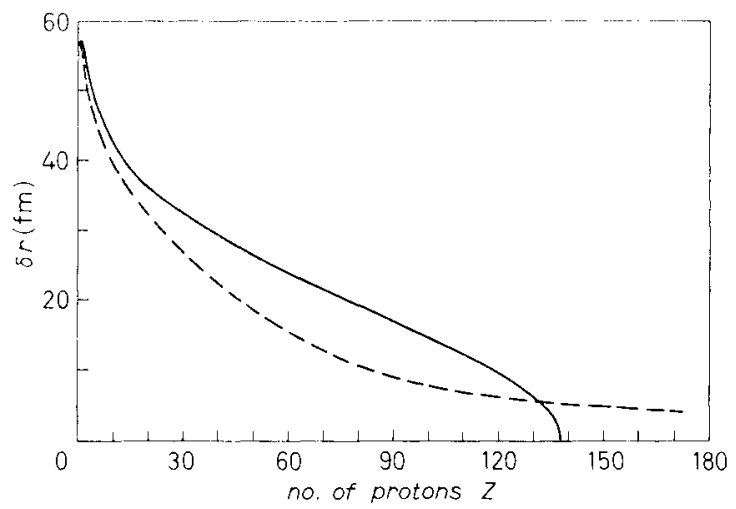

Fig. 1. - The "zitterbewegung "amplitude $\delta r$ as function of $/ x$. The solid line represents $\delta r$ aceording to BJokKex and DReLL $\left.{ }^{6}\right)$. The values given by the dashed line lead to the same results for the vacum fluctuation as the exact calculations of Desmovio ot $a l$. $\left.{ }^{1}\right)$. For $Z>90$ the dashed line is continued for large $Z$.

dition to this is a computer program which integrates the Dirac equation numerically (") - with any given potential, so that the effect of the extended nucleus and, if needed, the potential from the other electrons in the atom can be included. To introduce now the "zitterbewegung" we folded the potential with a Gaussian $\left.f\left(\left|\boldsymbol{r}^{\prime}\right|\right)=\exp \left|-\boldsymbol{r}^{\prime 2}\right|\left\langle(\delta r)^{2}\right\rangle\right]$ having a half-width $\delta r$, so that one gets a new potential by calculating

$$
V_{\text {new }}(\boldsymbol{r})=\frac{\int \mathrm{d} \boldsymbol{r}^{\prime} V_{\text {old }}\left(\boldsymbol{r}+\boldsymbol{r}^{\prime}\right) f\left(\left|\boldsymbol{r}^{\prime}\right|\right)}{\int \mathrm{d} \boldsymbol{r}^{\prime} f\left(\left|\boldsymbol{r}^{\prime}\right|\right)}
$$

In doing this, each point in the electron wave function is distributed over a range $\delta r$, so that the potential really acting is modified. In an atom with a single electron this leads only to changes in the vicinity of the nucleus where the potential deviates from the $1 / r$ behavior. This is an expression of the fact that the vacumm fluctuation can be written as an effective potential aeting only at the point of the nueleus.

(i) J. D. BJonkex and S. D. DRELL: Relativistic Quntum Mechomics.

(7) We would like to thank Prof. J. T. WABER for communicating this computer program. 
In Table I the "exact " values for fluctuation $\left({ }^{2}\right)$ are eompared with the values of onr simple folding method. The agreement is astonishingly good in the region of small $Z$. For high $Z$ our values lie about a factor two too high above the calculation of DESIDERIO et al. $\left({ }^{4}\right)$ but very near to the value of Brown et al. $\left({ }^{5}\right)$.

This result slows that the old quite simple explanation of fluctuation leads to reasonable results and is a practical method for calculation of the shifts of the s levels, where the ceffect of fluctuation is most strongly (for example, in $\mathrm{H}$ the $2 s$ shift is $1070 \mathrm{M} \mathrm{Hz}$, whereas the $2 p_{1}$ shift is only -- $\left.12 \mathrm{IH} \mathrm{H}\right)$. Because of $\mathrm{l}$-dependent influences, the simple folding method is not nseful for levels with $l \neq 0$. The folded new potential dif-

TABie I. - The varuum flutuation arconding to the simple folding wethod in comparison to the olssered, respertively, calculated ralues for ravious s levels in electronir atoms.

\begin{tabular}{|c|c|c|c|}
\hline \multirow[t]{2}{*}{ Elenent } & \multirow[t]{2}{*}{ Ievel } & \multicolumn{2}{|l|}{ lacuun fluctuation } \\
\hline & & (xact calculation $\left(^{*}\right)$ & present calculation \\
\hline \multirow[t]{3}{*}{ II } & $\mathrm{I} s$ & $8.55 \mathrm{GHz}$ & $8.59 \mathrm{CHz}$ \\
\hline & $2 s$ & $1.07 \mathrm{CH} \%$ & $1.08 \mathrm{GII}$ \\
\hline & $3 x$ & $0.31 \mathrm{GHz}$ & $0.31 \mathrm{GHz}$ \\
\hline \multirow[t]{3}{*}{$11 a^{-!}$} & $2 x$ & $14.27 \mathrm{GHz}$ & $14.45 \mathrm{C}_{x} \mathrm{~Hz}$ \\
\hline & $3 x$ & $4.25 \mathrm{GH} \%$ & $4.34 \mathrm{GHz}$ \\
\hline & $4 s$ & $\mathrm{I} .79 \mathrm{GHz}$ & $1.81 \mathrm{GHz}$ \\
\hline $\mathrm{L}^{2} \mathrm{i}^{\mathrm{i}}$ & $2 s$ & $64.0 \mathrm{GHz}$ & $65.0 \mathrm{GHz}$ \\
\hline$(5.5)$ & $2 \leqslant$ & $770.0 \quad(\mathrm{xH} \%$ & $820.0 \mathrm{GHz}$ \\
\hline \multirow[t]{2}{*}{$Z=-=70$} & $1 ;$ & 9.1 Ryd & $22.2 \mathrm{Ryd}$ \\
\hline & $2 s$ & & $3.2 \mathrm{Ryd}$ \\
\hline \multirow[t]{2}{*}{$Z=80$} & $1 \%$ & 15.0 Ryd & 39.0 Ryd \\
\hline & $2 s$ & & 6.4 Ryd \\
\hline \multirow[t]{2}{*}{$7 x=90$} & $1 s$ & $23.5 \mathrm{R}$ Rd & $66.8 \mathrm{Ryd}$ \\
\hline & $2 s$ & & $11.2 \mathrm{Ryd}$ \\
\hline
\end{tabular}

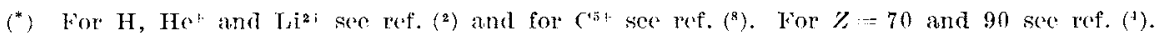

TAses II. -.. The racuum flustuation sontribution in muonic atoms from calsulations of BAREET $\left({ }^{9}\right)$ in comparison to the present folding method.

\begin{tabular}{|c|c|c|c|}
\hline \multirow[t]{2}{*}{ Element } & \multirow[t]{2}{*}{ Ixtel } & \multicolumn{2}{|l|}{ Vacuum fluctuation } \\
\hline & & alculation of BARRETT $\left({ }^{8}\right)$ & present caleulation \\
\hline \multirow[t]{2}{*}{ ( ia } & $1 s$ & $0.21 \mathrm{kel}$ & $0.20 \mathrm{kel}$ \\
\hline & $2 s$ & $0.05 \mathrm{Ke} / \mathrm{V}$ & $0.03 \mathrm{kel}$ \\
\hline \multirow[t]{2}{*}{ TY } & Is & $3.17 \mathrm{keV}$ & $0.78 \mathrm{kel}$ \\
\hline & $2 s$ & $0.74 \mathrm{ke}^{\circ}$ & $0.42 \mathrm{keV}$ \\
\hline
\end{tabular}

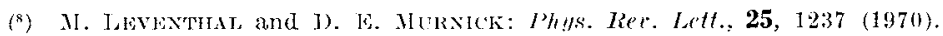

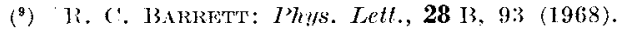


fers very much from the old potential especially at the origin, so that the effect belonging to this change cannot be calculated by ordinary perturbation theory. A totally new integration with the new potential has to be done.

In Table II the calculation of vacuum fluctuation with this method in muonic atoms is compared with the calculations of BARRETT $\left({ }^{9}\right)$. We get results of the same order of magnitude that they get.

\section{3. - Extrapolation to superheavy elements.}

The values for the fluctuation of the 18 levels in the elements $Z=70 \div 90$ calculated by DEsIDERIO et al. $\left({ }^{4}\right)$ gives us the possibility to determine a curve with new $\delta r$ values for high- $Z$ elements, which lead to the same results. This curve is given in Fig. 1 as a dashed line. To get an idea of how large the effect of fluctuation will be for very high $Z$, we continued this dashed line and calculated first-approximation values for vacuum fluctuation for elements up to $Z=180$, where this effect becomes large. The results are shown in Fig. 2.

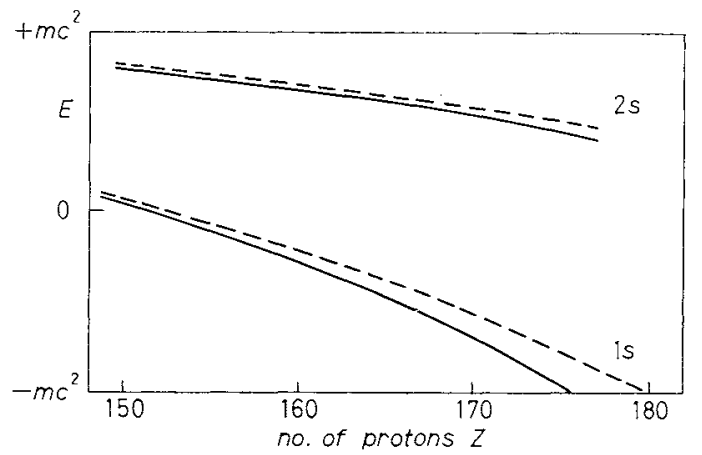

lig. 2. - The solid lines give the energy eigenvalues for the 18 and $2 \mathrm{~s}$ levels from self-consistent calctilalions for elements between $Z=150$ and $Z==180$. The dashed lines are the energy eigenvalues including the vacuum fluctuation contribution.

Nobody knows up to now how large the effects of quantum electrodynamics will be for elements with $Z>137$, but one expects that the energy eigenvalnes for the inner electrons do not reach the continuun of electrons with negative energy $\left({ }^{10}\right)$. Therefore, this simple folding method might be quite a good description of the behavior of inner electrons in superheavy elements. The decision how large the quantum electrodynamical effects really are has to be given by an exact calculation. This theory of quantum clectrodynamies of strong fields is in progress by REINInRDT et al. (i1). The exact calculation will be most complicated, so that in practical cases the calculation given in this letter will lead to the right order of magnitude in this simple way which will be sufficient in many cases.

I would like to thank Profs. J. T. Waber and W. Greiner for their great interest and several discussions.

(10) W. Grinen: Panel Discussion (Hontreal, 1969).

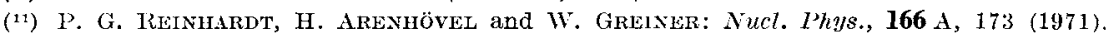

